responsible for the loss of 42000 lives in 2016. Among the stressful professions that endure a high consumption of cigarettes per day, are the stressful professions related to television work: producers, editors, reporters, picture directors. This paper aims to present the tobacco cessation program developed in National Romanian Television in order to promote the health of its employees and to reduce the number of smoking related illnesses.

Methods We started to achieve the present work in the current legislative context, in which the adoption of the Antismoking Law in 2016, prohibited smoking in the public spaces, so also in the spaces of National Romanian Television. That is why we have structured, since February 1, 2017, a Tobacco Cessation Program, by professional categories, insisting on the VBA (Very Brief Advise) but also on the pharmacological methods of quitting (nicotine replacement therapy, varenicline, bupropion).

Result Smoking cessation results were satisfactory in the group of employees with VBA associated with pharmacological therapy $(27.5 \%$ quit smoking and $25.3 \%$ reduced to more than half the number of cigarettes smoked in one day). The tobacco cessation program will continue, requiring the inclusion of all professional categories involved in television work and will be targeted especially on employees with chronic diseases (COPD, asthma, hypertension, chronic ischaemic heart disease, diabetes).

Discussion Smoking cessation is an essential method of promoting health at work and decreasing the number of chronic illnesses and deaths attributable to smoking. We propose in the future the development of the program and the inclusion of behavioural support for smoking cessation as well as CO monitoring in expired air.

\section{SICK LEAVE BENEFITS BY ENDOCRINE DISEASES: A RETROSPECTIVE EPIDEMIOLOGICAL STUDY}

LFT Priester*, DVS Vitor, RV de Brito, DVS Vitor, LR Ferreira. Centro Universitário das Faculdades Associadas de Ensino, São João da Boa Vista, Brazil

\subsection{6/oemed-2018-ICOHabstracts.466}

Introduction Endocrine Diseases (ED) have a high prevalence around the world. In 2014, the World Health Organisation estimated that many adults will be affected by thoses diseases, worldwide. ED are classified as many different chronic diseases with distinct aetiologies, and they are some of the main causes of disability, which can negatively influence productivity in active workers. In Brazil, those disabled workers with ED diseases can apply for Sick Leave Benefits granted by the Brazilian National Institute of Social Security (BNISS). This Institute is responsible for granting benefits and salaries for work disability.

Methods This was a retrospective cross-sectional study which evaluated 538 workers receiving temporary sick leave benefits from the BNISS caused by ED. The workers were evaluated from June 2014 until July 2016 and submitted to a structured analysis for data collection including: gender, age, affiliation to the BNISS and International Classification of Diseases (ICD-10) of each worker's ED.

Results The workers were $63,5 \%$ female with mean age of 42 years-old, and the most prevalent individuals age ranged from 30 to 49 years old (44\%). The most prevalent diseases were associated with obesity (33\%) with ICD-10 E65 and E66, followed by Diabetes $(23,5 \%)$ with ICD-10 E10, E11 and E14, and thyroid associated diseases (7,8\%) with codes E01, E03, E04, E05, E06, E07. The employed affiliation to BNISS was found in $66,5 \%$ of females, and $50 \%$ of males. Other endocrine diseases were found, as amiloidosis (0,2\%) ICD-10 E85, and other endocrine disturbs (4\%) ICD-10 E88.

Discussion The results showed that obesity and diabetes are important risks factors for functional endocrine injuries, enhancing the harmful effects of overweight and hiperglicemic diet to health and quality of life of workers. These data can help new strategies to enhance obesity and diabetes prevention and health promotion programs for workers.

\section{OCCUPATIONAL HEALTH NURSES WORKING AS WORKSITE HEALTH PROMOTION AGENTS}

${ }^{1}$ Pablo Zarate, 'Diego Cuellar, 'Luis Velazquez, ${ }^{2}$ Leila Cura*. ${ }^{1}$ Pan American Energy, Comodoro Rivadavia, Argentina; ${ }^{2}$ Pan American Energy, Buenos Aires, Argentina

\subsection{6/oemed-2018-ICOHabstracts.467}

Introduction Traditional role in occupational nursing had been for many years to assist occupational physicians and provide primary health care services. Worksite health promotion field requires a different role for occupational nurses. Our team was trained in health coaching and started offering a new service for workers. Workers can have their personal coach for lifestyle risk management. In this poster we selected two experiences to communicate the impact of this kind of recourse for worksite health promotion programs.

Methods In an Company a 1000 employees from the oil and gas industry with 98\% of workers screened for lifestyle risks annually, we define as target of the intervention 2 major risks: Hypertension and weight issues. Two wellness programs were offered: Blood pressure initiative and Wise. Occupational Nurses were the health promotion agents in charge of the program implementation. We measure each risk at the beginning and we measure impart of the intervention. Core intervention were health coaching interactions.

Result Wise program 219 enrolled employees, 182 participating, Initial measures: overweight: $36.3 \%$, obese type 1: $33.0 \%$, obese type $2: 16.5 \%$, obese type $3: 4.9 \%$, healthy weight: $9.3 \%$. Final measures: overweight: $37.9 \%$, obese type $1: 34.6 \%$, obese type 2: $12.6 \%$, obese type 3: 4.9\%, healthy weight: 9.9\%.: $71 \%$. Total weight loss: $484.90 \mathrm{~kg}$. Blood pressure program: 935 enrolled and screened employees, 184 employees didn't knew they had hypertension measurements and of them 98 were diagnosed. Healthy habits modification: $71 \%$.

Conclusion Health coaching is a powerful intervention for healthy habits modification in order to reduce lifestyle related risks. Occupational nurses are a strategic group of health professionals for worksite health promotion programs. All this aspects may contribute to develop new roles for occupational health nurses to contribute to build a healthy culture in the workplace.

\section{COST OF PROVIDING OCCUPATIONAL HEALTH SERVICE FOR INFORMAL WORKERS AT SUB-DISTRICT HEALTH PROMOTING HOSPITAL LEVEL}

${ }^{1}$ Simalak Dithisawatwet* ${ }^{2}$ Songsab Pimchainoi, ${ }^{3}$ Sasithorn Thanapob. ${ }^{1}$ The Office of Disease Prevention and Control 8 Udonthani; ${ }^{2}$ The Office of Disease Prevention and Control 7 Khonkaen; ${ }^{3}$ School of Public Health, Walailak University

10.1136/oemed-2018-ICOHabstracts.468 